\title{
The Hadamard circulant conjecture
}

\author{
Barry Hurley, Paul Hurley and Ted Hurley
}

\section{Abstract}

It is shown that if $H$ is a circulant Hadamard $4 n \times 4 n$ then $n=1$. This proves the Hadamard circulant conjecture.

\section{Introduction}

A Hadamard matrix $H$ of order $m$ is an $m \times m$ matrix with entries \pm 1 such that $H H^{\mathrm{T}}=$ $m I_{m}$ where $I_{m}$ is the identity $m \times m$ matrix. Hadamard matrices only exist when $m=1,2$ or $m$ is a multiple of 4 . They have many applications, including in coding theory, cryptography and signal processing (details of which may be found in [1] or elsewhere).

The Hadamard conjecture states that there is a Hadamard $4 n \times 4 n$ matrix for every $n$. For background information on this conjecture, we refer the reader to $\mathbf{1}$ and the references therein. The first unknown case at present is $m=668$, the previous unknown case $m=428$ being solved in $[3]$.

A circulant matrix is a matrix whose rows are cycle permutations as follows:

$$
\left(\begin{array}{ccccc}
a_{0} & a_{1} & a_{2} & \ldots & a_{t-1} \\
a_{t-1} & a_{0} & a_{1} & \ldots & a_{t-2} \\
\vdots & \vdots & \vdots & \vdots & \vdots \\
a_{1} & a_{2} & \ldots & a_{t-1} & a_{0}
\end{array}\right)
$$

and a circulant Hadamard matrix is when it is additionally Hadamard.

A $4 \times 4$ Hadamard circulant matrix is one with first row containing three elements of the same sign and another element of a different sign, as for example in:

$$
\left(\begin{array}{cccc}
1 & 1 & 1 & -1 \\
-1 & 1 & 1 & 1 \\
1 & -1 & 1 & 1 \\
1 & 1 & -1 & 1
\end{array}\right)
$$

We prove the circulant Hadamard matrix conjecture, namely, that circulant Hadamard $4 n \times 4 n$ matrices do not exist for $n>1$.

Theorem 1.1. Let $H$ be an $m \times m$ Hadamard circulant matrix. Then $m=1$ or $m=4$.

2000 Mathematics Subject Classification 00000.

2000 Mathematics Subject Classification: 15B34, 16S34. 
The Hadamard circulant conjecture appears to have been mentioned first in a book by Ryser [5] but goes back further to obscure sources. What was known about their existence includes:

- Turyn $[9$ proved that no circulant Hadamard matrices of orders $8 p$ exist and excluded other orders of form $4(p+1)$.

- A Hadamard circulant matrix must either have order $m=4 n^{2}$ or $m=1$ [7, 8 .

- There is no Hadamard circulant matrix of order $n$ with $4<n \leq 10^{11}$ with three possible exceptions [6, 7].

For connections between Ryser's conjecture, the circulant Hadamard matrix conjecture and Barker's conjecture see the monograph [8] or paper $\mathbf{7}$.

\subsection{Outline}

Section 2 provides the necessary background for the proof, which makes use of the connection between the group ring of the cyclic group and circulant matrices. When the multiplicative cyclic group $C_{m}$ with generator $g$ is listed in the 'natural' order, namely $\left\{1, g, g^{2}, \ldots, g^{m-1}\right\}$, a circulant matrix is obtained.

When a different listing is used this reduces the problem to working with certain block matrices. A different listing changes the corresponding matrix by interchanging rows and columns and thus if the original matrix is Hadamard the matrix relative to the new listing is also Hadamard. This new listing can be seen in retrospect to be more 'natural' and shows why a circulant $m \times m$ Hadamard matrix when $m>4$ cannot exist.

As we explain later, they fail to be Hadamard because of a certain 'twist' that is introduced into the blocks formed. By avoiding this twist, Hadamard matrices from group ring elements may be formed. Using a listing in the group so that the corresponding matrix forms rows of $2 \times 2$ blocks seems to be more natural.

This idea also leads to examples of Hadamard almost circulant matrices (section 4).

The main part of the paper is the proof in section 3 .

The proof consists of the following steps:

- The re-derivation of the known result [7, 8, that $n$ must be a perfect square is initially derived.

- The elements of $C_{4 n}$ are listed $\{0,2 n, 1,2 n+1, \ldots, 2 n-1,4 n-1\}$ where $i$ denotes $g^{i}$. Now form from the group ring $\mathbb{Z} C_{4 n}$ the (Hadamard) $R G$-matrix relative to this listing.

- This results in blocks $\left(\begin{array}{cc}i & 2 n+i \\ 2 n+i & i\end{array}\right)$ which are either even $\left(\begin{array}{c}++ \\ +\end{array}\right),\left(\begin{array}{l}-- \\ --\end{array}\right)$ or odd $\left(\begin{array}{l}+- \\ -\end{array}\right)$, $\left(-_{+}^{+}\right)$. There are then an equal number of even and odd blocks.

- For the matrix to be Hadamard the odd blocks must occur in matching cancelling pairs; this is shown to be impossible for $n>1$.

The natural question to then ask is if Hadamard matrices can arise from other group rings. Thus, in section 4 , we provide examples of such matrices. These are formed within the group ring of $\mathbb{Z}\left(C_{2} \times C_{8}\right)$ and within the group ring $\mathbb{Z}\left(\mathbb{H} \times C_{2}\right)$, where $C_{t}$ denotes the cyclic group of order $t$ and $\mathbb{H}$ is the quaternion group of order 8 . These can be extended to give Hadamard group ring matrices over $\mathbb{Z}\left(C_{2} \times C_{8} \times C_{4}^{t}\right)$ and over $\mathbb{Z}\left(\mathbb{H} \times C_{2} \times C_{4}^{t}\right)$ for any $t$. $C_{4}$ can be replaced by $C_{2} \times C_{2}$ to give similar examples.

\section{Background}

In this section, we introduce the necessary background in group rings, and related matrices. 


\subsection{Group rings and matrices}

Let $R$ denote a ring and $G$ a group. The group ring $R G$ consists of all (finite support) $\sum_{g_{i} \in G} \alpha_{g_{i}} g_{i}$ with $\alpha_{g_{i}} \in R$. For further details on group rings see for example [4].

Let $G=\left\{g_{1}, g_{2}, \ldots, g_{n}\right\}$ denote the elements of a group $G$ of order $n$ and consider the elements of $G$ as being listed in this particular order. We shall be particularly interested in the case when $G$ is a cyclic group of order $n$ generated by $g$. The matrix of $G$, called the $G$-matrix, (see for example [2]), relative to this listing is

$$
\left(\begin{array}{ccccc}
g_{1}^{-1} g_{1} & g_{1}^{-1} g_{2} & g_{1}^{-1} g_{3} & \ldots & g_{1}^{-1} g_{n} \\
g_{2}^{-1} g_{1} & g_{2}^{-1} g_{2} & g_{2}^{-1} g_{3} & \ldots & g_{2}^{-1} g_{n} \\
\vdots & \vdots & \vdots & \vdots & \vdots \\
g_{n}^{-1} g_{1} & g_{n}^{-1} g_{2} & g_{n}^{-1} g_{3} & \ldots & g_{n}^{-1} g_{n}
\end{array}\right)
$$

Suppose then $w=\sum_{i=1}^{n} \alpha_{g_{i}} g_{i} \in R G$. Then

$$
w \mapsto\left(\begin{array}{ccccc}
\alpha_{g_{1}^{-1} g_{1}} & \alpha_{g_{1}^{-1} g_{2}} & \alpha_{g_{1}^{-1} g_{3}} & \ldots & \alpha_{g_{1}^{-1} g_{n}} \\
\alpha_{g_{2}^{-1} g_{1}} & \alpha_{g_{2}^{-1} g_{2}} & \alpha_{g_{2}^{-1} g_{3}} & \ldots & \alpha_{g_{2}^{-1} g_{n}} \\
\vdots & \vdots & \vdots & \vdots & \vdots \\
\alpha_{g_{n}^{-1} g_{1}} & \alpha_{g_{n}^{-1} g_{2}} & \alpha_{g_{n}^{-1} g_{3}} & \ldots & \alpha_{g_{n}^{-1} g_{n}}
\end{array}\right)
$$

gives an embedding of $R G$ into the ring of $n \times n$ matrices over $R$ relative to this listing of $G$.

Call the above matrix the $R G$-matrix of $w$ relative to the given listing and call a matrix obtained from the group ring $R G$ in this manner an $R G$-matrix. The term $R G$-matrix is used here as the methods are basically group ring methods.

The structure of an $R G$-matrix may clearly be seen in the following table:

$$
\left(\begin{array}{l|lllll} 
& g_{1} & g_{2} & g_{3} & \ldots & g_{n} \\
\hline g_{1}^{-1} & \alpha_{g_{1}^{-1} g_{1}} & \alpha_{g_{1}^{-1} g_{2}} & \alpha_{g_{1}^{-1} g_{3}} & \ldots & \alpha_{g_{1}^{-1} g_{n}} \\
g_{2}^{-1} & \alpha_{g_{2}^{-1} g_{1}} & \alpha_{g_{2}^{-1} g_{2}} & \alpha_{g_{2}^{-1} g_{3}} & \ldots & \alpha_{g_{2}^{-1} g_{n}} \\
\vdots & \vdots & \vdots & \vdots & \vdots & \vdots \\
g_{n}^{-1} & \alpha_{g_{n}^{-1} g_{1}} & \alpha_{g_{n}^{-1} g_{2}} & \alpha_{g_{n}^{-1} g_{3}} & \ldots & \alpha_{g_{n}^{-1} g_{n}}
\end{array}\right)
$$

The first column is essentially labelled by $g_{1}$, the second by $g_{2}$ etc.; the first row is labelled by $g_{1}^{-1}$, the second by $g_{2}^{-2}$ etc. As it aids in the calculation of an $R G$-matrix relevant to a particular listing, this formulation will be called upon later.

2.1.1. Cyclic group rings, circulant matrices, and alternate listings. When $G=C_{n}=$ $\left\{1, g, g^{2}, \ldots, g^{n-1}\right\}$ is the cyclic group of order $n$, we get a group ring $R C_{n}$.

Let $R C_{n \times n}$ denote the ring of circulant $n \times n$ matrices which is a subring of $R_{n \times n}$, the ring of $n \times n$ matrices over $R$.

Then $R C_{n \times n} \cong R C_{n} \cong \frac{R[x]}{\left\langle x^{n}-1\right\rangle}$ where $R[x]$ is the polynomial ring in variable $x$ over $R$ and $\left\langle x^{n}-1\right\rangle$ is the ideal in $R[x]$ generated by $x^{n}-1$.

The isomorphism makes it possible to move from the circulant matrices to the cyclic group ring and back, exploiting properties of both. 
Let $w=\sum_{i=0}^{n-1} \alpha_{i} g^{i}$. Then the $R G$-matrix of $w$ relative to this natural listing is the circulant matrix

$$
\left(\begin{array}{ccccc}
\alpha_{0} & \alpha_{1} & \alpha_{2} & \ldots & \alpha_{n-1} \\
\alpha_{n-1} & \alpha_{0} & \alpha_{1} & \ldots & \alpha_{n-2} \\
\vdots & \vdots & \vdots & \vdots & \vdots \\
\alpha_{1} & \alpha_{2} & \ldots & a_{n-1} & \alpha_{0}
\end{array}\right)
$$

Suppose, in the general case, the listing is changed. Any change of listing corresponds to a permutation of the elements of the group and a permutation is generated by transpositions. If the elements $g_{i}$ and $g_{j}$ are interchanged then the new matrix is obtained from the original matrix by interchanging the $i^{t h}$ and $j^{\text {th }}$ rows and then interchanging the $i^{\text {th }}$ and $j^{\text {th }}$ columns. Thus if an $R G$-matrix relative to some listing is Hadamard then the $R G$-matrix relative to any listing, as one might expect, is also Hadamard.

For example, let $G$ be the cyclic group $C_{4}$ generated by $g$ with $w=1+g+g^{2}-g^{3}$.

Then relative to the natural ordering $w$ has the Hadamard circulant matrix $\left(\begin{array}{cccc}1 & 1 & 1 & -1 \\ -1 & 1 & 1 & 1 \\ 1 & -1 & 1 & 1 \\ 1 & 1 & -1 & 1\end{array}\right)$.

When $G$ has the listing $G=\left\{1, g^{2}, g, g^{3}\right\}, w$ has the Hadamard matrix $\left(\begin{array}{cc|cc}1 & 1 & 1 & -1 \\ 1 & 1 & -1 & 1 \\ \hline-1 & 1 & 1 & 1 \\ 1 & -1 & 1 & 1\end{array}\right)$.

The new listing is obtained by interchanging $g$ and $g^{2}$ in the original listing, and thus the new matrix is obtained from the original by interchanging the second and third rows and then the second and third columns. Notice how the 'odd' block $\left(\begin{array}{cc}1 & -1 \\ -1 & 1\end{array}\right)$ of this new matrix gets 'twisted' to a new type of 'odd' block $\left(\begin{array}{cc}-1 & 1 \\ 1 & -1\end{array}\right)$ in the next row of blocks as indicated; in this small case however this is not a problem as the even block still acts above and below the 'odd' blocks.

\section{Proof of the conjecture}

Suppose now that $H$ is a circulant Hadamard $4 n \times 4 n$ matrix. Let $C_{4 n}$ be generated by $g$ and let $w$ be the element in the cyclic group ring $\mathbb{Z} C_{4 n}$ corresponding to $H$. The inner product of any two (different) rows of a Hadamard matrix is 0.

\section{1. $n$ must be a perfect square.}

Suppose there are $r$ negative entries in the first row of $H$ and consequently there are $r$ negative elements in each row and column. There are then $(4 n-r)$ positive entries in each row and column.

It is known that for any existing circulant $4 n \times 4 n$ Hadamard matrix $n$ must be a perfect square [7, 8. For completeness, the following verifies this result and additionally provides information on the number of possible negative and positive entries.

LEMMA 3.1. $r=2 n \pm \sqrt{n}$.

Proof. The sum of the elements in any column is $4 n-r-r=4 n-2 r$.

The sum of the elements in a column except a positive element is thus $4 n-2 r-1$ and the sum of the elements in a column except a negative element is $4 n-2 r+1$. The rows of the matrix are orthogonal to one another and thus the sum of the first $4 n-1$ rows is perpendicular 
to the last row. The last row has $4 n-r$ positive elements and $r$ negative elements. Thus $(4 n-r)(4 n-2 r-1)-r(4 n-2 r+1)=0$.

This implies that

$$
16 n^{2}-8 n r-4 n-4 n r+2 r^{2}+r-4 n r+2 r^{2}-r=0 .
$$

Hence $16 n^{2}-16 n r+4 r^{2}-4 n=0$ from which $r^{2}-4 n r+4 n^{2}-n=0$. Solving this quadratic for $r$ gives:

$$
r=\frac{4 n \pm \sqrt{16 n^{2}-16 n^{2}+4 n}}{2}
$$

and so $r=2 n \pm \sqrt{n}$, as required.

It then follows that the number of positive elements is $4 n-r=2 n \mp \sqrt{n}$, as expected from symmetry.

The result of Lemma 3.1 depends only on the fact that the same number of positive elements appears in each row and column. A Hadamard matrix whose row and column sums are the same is known as a regular Hadamard matrix. Thus whenever $R G$-matrices (which include circulant matrices) are Hadamard, they are by necessity also regular Hadamard matrices.

The following may be proved along the lines of Lemma 3.1

Lemma 3.2. Let $A$ be a $m \times m$ matrix with orthogonal rows consisting of \pm 1 entries in which the same number $m-r$ of +1 and the same number, $r$, of -1 appear in each row and column. Then $r=\frac{m \pm \sqrt{m}}{2}$.

\subsection{New listing: Pairs in blocks}

The elements of the cyclic group $G=C_{4 n}$ are $\left\{1, g, g^{2}, \ldots, g^{4 n-1}\right\}$, listed in the 'natural' ordering. Write $i$ for $g^{i}$ so this listing is $\{0,1,2, \ldots, 4 n-1\}$.

Now $H$ is a Hadamard circulant matrix obtained from the $R G$-matrix corresponding to an element of $\mathbb{Z} G$ relative to this natural ordering. An interchange of rows or columns in a Hadamard matrix results in another Hadamard matrix. If two group elements are interchanged in the listing then the resulting $R G$-matrix is obtained by interchanging two rows and then interchanging two columns and the result is thus a Hadamard matrix. Any new listing may be obtained by a succession of interchanges and thus if the listing is changed the new $R G$-matrix obtained is still a Hadamard matrix.

List the elements of $G$ as follows: $\{0,2 n, 1,2 n+1, \ldots, 2 n-1,4 n-1\}$. Using this ordering breaks the $R G$-matrix into blocks of $2 \times 2$ matrices and rows of these blocks. The inner product of any two (different) rows is 0 as the matrix is Hadamard.

Let $\widetilde{i}$ denote the coefficient of $g^{i}$ in the $R G$-matrix; each $\widetilde{i}$ is either +1 or -1 .

Consider now the $R G$-matrix with this listing as constructed in (2.1) of Section 2.1. The first two rows are then:

$$
\left(\begin{array}{cccccccc}
\widetilde{0} & \widetilde{2 n} & \widetilde{1} & \widetilde{2 n+1} & \ldots & \ldots & \widetilde{2 n-1} & \widetilde{4 n-1} \\
\widetilde{2 n} & \widetilde{0} & \widetilde{2 n+1} & \widetilde{1} & \ldots & \ldots & \widetilde{4 n-1} & \widetilde{2 n-1}
\end{array}\right)
$$

The inner product of these two rows gives that $2\{(\widetilde{0} \times \widetilde{2 n})+\widetilde{1} \times(\widetilde{(2 n+1})+\widetilde{2} \times(\widetilde{2 n+2})+\ldots+$ $(\widetilde{2 n-1}) \times(\widetilde{4 n-1}))\}=0$. Hence $\widetilde{0} \times \widetilde{2 n}+\widetilde{1} \times(\widetilde{2 n+1})+\widetilde{2} \times(\widetilde{2 n+2})+\ldots+(\widetilde{2 n-1}) \times(\widetilde{4 n-1})=$ 0 . 
Thus one half of the products $\tilde{i} \times(\widetilde{2 n+i}), 0 \leq i \leq 2 n-1$, are +1 and one half are -1 . Hence $\widetilde{i}, \widetilde{2 n+i})$ for $i=0, \ldots 2 n-1$ consist of $(+1,-1)$ or $(-1,+1)$ for $n$ times and $(+1,+1)$ or $(-1,-1)$ for the other $n$ times.

Consider in more detail the $G$-matrix obtained from the listing $\{0,2 n, 1,2 n+1, \ldots, 2 n-$ $1,4 n-1\}$

$$
\left(\begin{array}{cccccccccc}
0 & 2 n & 1 & 2 n+1 & 2 & 2 n+2 & \ldots & \ldots & 2 n-1 & 4 n-1 \\
2 n & 0 & 2 n+1 & 1 & 2 n+2 & 2 & \ldots & \ldots & 4 n-1 & 2 n-1 \\
\hline 4 n-1 & 2 n-1 & 0 & 2 n & 1 & 2 n+1 & \ldots & \ldots & 2 n-3 & 4 n-3 \\
2 n-1 & 4 n-1 & 2 n & 0 & 2 n+1 & 1 & \ldots & \ldots & 4 n-3 & 2 n-3 \\
\hline \vdots & \vdots & \vdots & \vdots & \vdots & \vdots & \vdots & \vdots & \vdots & \vdots
\end{array}\right)
$$

The elements of $G$ are replaced by their coefficients to obtain the $R G$-matrix which is assumed to be Hadamard.

It is convenient to write $i$ for the coefficient $\widetilde{i}$ of $i=g^{i}$ in the $R G$ matrix. Thus when considering the $G$-matrix $i=g^{i}$, and when considering the $R G$-matrix $i$ denotes the coefficient of $g^{i}$.

For example on considering the above matrix as an $R G$-matrix the inner product of the first row with the third row gives that

$$
(0,2 n) \cdot(4 n-1,2 n-1)+(1,2 n+1) \cdot(0,2 n)+\ldots+(2 n-1,4 n-1) \cdot(2 n-3,4 n-3)=0
$$

where $\cdot$ denotes the inner product of the length 2 vectors.

The $R G$-matrix formed - see (2.1) above - with this listing is now considered in more detail. The matrix has been broken into $2 \times 2$ blocks for detailed analysis. Here $i$ in the first row or column means the group element $g^{i}$, and $i$ elsewhere is the coefficient of $g^{i}$ which is either +1 or -1 .

\begin{tabular}{c||cc|cc|cc|c|cc} 
& 0 & $2 n$ & 1 & $2 n+1$ & 2 & $2 n+2$ & $\ldots$ & $2 n-1$ & $4 n-1$ \\
\hline \hline 0 & 0 & $2 n$ & 1 & $2 n+1$ & 2 & $2 n+2$ & $\ldots$ & $2 n-1$ & $4 n-1$ \\
$2 n$ & $2 n$ & 0 & $2 n+1$ & 1 & $2 n+2$ & 2 & $\ldots$ & $4 n-1$ & $2 n-1$ \\
\hline $4 n-1$ & $4 n-1$ & $2 n-1$ & 0 & $2 n$ & 1 & $2 n+1$ & $\ldots$ & $2 n-2$ & $4 n-2$ \\
$2 n-1$ & $2 n-1$ & $4 n-1$ & $2 n$ & 0 & $2 n+1$ & 1 & $\ldots$ & $4 n-2$ & $2 n-2$ \\
\hline$\vdots$ & $\vdots$ & $\vdots$ & $\vdots$ & $\vdots$ & $\vdots$ & $\vdots$ & $\vdots$ & $\vdots$ & $\vdots$ \\
\hline $2 n+1$ & $2 n+1$ & 1 & $2 n+2$ & 2 & $2 n+3$ & 3 & $\ldots$ & 0 & $2 n$ \\
1 & 1 & $2 n+1$ & 2 & $2 n+2$ & 3 & $2 n+3$ & $\ldots$ & $2 n$ & 0 \\
\hline
\end{tabular}

Notice that the last block in a row of blocks is 'twisted' when it appears as the first block of the next row of blocks, as for example $\left(\begin{array}{cc}2 n-1 & 4 n-1 \\ 4 n-1 & 2 n-1\end{array}\right)$ in the first row of blocks becomes $\left(\begin{array}{ll}4 n-1 & 2 n-1 \\ 2 n-1 & 4 n-1\end{array}\right)$ in the second row of blocks.

The inner product of any two rows must all be 0 since the matrix is Hadamard. The inner product of a row of a block with another row of that block has ensured that there are an equal number of even and odd pairs in each row of blocks.

\subsection{Even and odd blocks}

Suppose $B=\left(\begin{array}{cc}i & j \\ j & i\end{array}\right)$ where $i= \pm 1, j= \pm 1$. Call such a $B$ a 2-block or simply a block when the size is clear. Say that $B$ is even when $i=j$ and odd when $i \neq j$. Define $\widetilde{B}=\left(\begin{array}{cc}j & i \\ i & j\end{array}\right)$. Thus $\widetilde{B}=-B$ when $B$ is odd and $\widetilde{B}=B$ when $B$ is even. 
Let $m=2 n$. The matrix has been broken into 2-blocks as follows:

$$
M=\left(\begin{array}{llllll}
\widetilde{B_{1}} & B_{2} & B_{3} & \ldots & \ldots & B_{m} \\
\widetilde{B_{m}} & \widetilde{B_{1}} & B_{2} & \ldots & \ldots & B_{m-1} \\
\widetilde{B_{m-1}} & \widetilde{B_{m}} & B_{1} & \ldots & \ldots & B_{m-2} \\
\vdots & \vdots & \vdots & \vdots & \vdots & \vdots \\
\widetilde{B_{2}} & \widetilde{B_{3}} & \widetilde{B_{4}} & \ldots & \widetilde{B_{m}} & B_{1}
\end{array}\right)
$$

The inner product of two rows of blocks of $M$ is the sum $\sum B_{i} B_{j}{ }^{\mathrm{T}}=\sum B_{i} B_{j}$ over each $B_{i}$ in one of the rows of blocks with its corresponding $B_{j}$ in the other row of blocks. As $M$ is Hadamard the inner product of two (different) rows of blocks in $M$ must be the zero matrix $0=0_{2 \times 2}$.

We can assume that $n \geq 4$ by Lemma 3.1 .

Now $B_{i} B_{j}=0$ when one of $B_{i}, B_{j}$ is even and the other is odd, $B_{i} B_{j}= \pm\left(\begin{array}{ll}2 & 2 \\ 2 & 2\end{array}\right)$ when both $B_{i}, B_{j}$ are even and $B_{i} B_{j}= \pm\left(\begin{array}{cc}2 & -2 \\ -2 & 2\end{array}\right)$ when both $B_{i}, B_{j}$ are odd. Thus in any sum of products of 2-blocks in an equation $\sum B_{i} B_{j}=0$ the part of the sum involving products between even 2-blocks and the part of the sum involving products between odd 2-blocks must both be 0 .

There are $n$ even blocks and $n$ odd blocks in the system $S=\left\{B_{1}, B_{2}, \ldots, B_{m}\right\}$ of 2-blocks of $M$. Denote block $B_{i}$ by $i$ when convenient and unambiguous. The blocks $i, j$ are said to be of the same kind if they are both even or both odd. Define the difference of $\left(B_{i}, B_{j}\right)$ to be $j-i$ for $i<j$ and to be $2 n-(j-i)$ for $i>j$. Call $\left(B_{j}, B_{i}\right)$ the conjugate of $\left(B_{i}, B_{j}\right)$. Denote the difference of $(i, j)$ by $d(i, j)$. The differences of $S$ consist of all differences $d(i, j)$ with $i, j \in S, i \neq j$.

Say the block $i$ is symmetric if there exists a block of the same kind at difference $n$ away, and otherwise say the block $i$ is not symmetric or is non-symmetric. When $i$ is symmetric denote by $i^{\prime}$ the block (of the same kind) satisfying $d\left(i, i^{\prime}\right)=n=d\left(i^{\prime}, i\right)$.

Define the sign of the even 2-block $\left(\begin{array}{ll}1 & 1 \\ 1 & 1\end{array}\right)$ to be +1 and define the sign of the other even 2 -block to be -1 . Define the sign of the odd 2-block $\left(\begin{array}{cc}1 & -1 \\ -1 & 1\end{array}\right)$ to be +1 and the sign of the other odd 2-block to be -1 .

Define the sign of a pair $(i, j)$ of blocks as follows. When $i, j$ are even, define the sign of $(i, j)$ to be +1 if both $i, j$ have the same sign and to be -1 otherwise. When $i, j$ are odd and $i<j$ define the sign of $(i, j)$ to be +1 if both $i, j$ have the same sign and to be -1 otherwise and define the sign $(j, i)$ (for $j>i$ ) to be the opposite to that of the sign of $(i, j)$. If any difference $t$ between odd 2-blocks occurs an odd number of times in differences of $S$ then the (inner) product of the first row of blocks of $M$ with the $(t+1)^{s t}$ row of blocks of $M$ cannot be 0 . Thus every difference between the odd blocks must occur an even number of times. Every pair of odd blocks must have a matching pair of odd blocks with equal difference and opposite sign. Similarly every difference between the even 2-blocks must occur an even number of times and every pair of even blocks must have a matching pair of even blocks with equal difference and opposite sign.

Consider now only pairs $(i, j)$ of blocks with $i \neq j$ where $i, j$ are of the same kind.

Let $i, j, k, l$ be blocks of the same kind with $i \neq j, k \neq l$. Then $d(i, j)=d(k, l)$ if and only if $d(j, i)=d(l, k)$. Say the pair of blocks $(i, j)$ is a match of the pair $(k, l)$ if and only if $d(i, j)=d(k, l)$ and the pairs have opposite signs; in this case say $(i, j)$ matches $(k, l)$ or that $(k, l)$ is a matching pair for $(i, j)$. Then $(i, j)$ matches $(k, l)$ if and only if $(j, i)$ matches $(l, k)$. Use $(i, j) \sim(k, l)$ to mean that $(i, j)$ matches $(k, l)$ and $(i, j) \nsim(k, l)$ to mean that $(i, j)$ does not match $(k, l)$. Every pair has a unique matching pair and say $(i, j)$ is matched with $(k, l)$ if $(i, j) \sim(k, l)$ match and $(i, j)$ has been uniquely assigned to $(k, l)$. It is implicitly assumed 
that if $(i, j)$ is matched with $(k, l)$ then the conjugates $(j, i)$ and $(l, k)$ are also matched with one another.

Let $i, j$ be symmetric odd blocks. Then $\left(i, i^{\prime}\right) \sim\left(i^{\prime}, i\right)$ and $\left(j, j^{\prime}\right) \sim\left(j^{\prime}, j\right)$. Also $d(i, j)=d\left(i^{\prime}, j^{\prime}\right)$ and $d\left(j, i^{\prime}\right)=d\left(j^{\prime}, i\right)$ and either $(i, j) \sim\left(i^{\prime}, j^{\prime}\right),(j, i) \sim\left(j^{\prime}, i^{\prime}\right)$ and $\left(j, i^{\prime}\right) \not$ $\left(j^{\prime}, i\right),\left(i^{\prime}, j\right) \nsim\left(i, j^{\prime}\right)$ or else $(i, j) \not\left(i^{\prime}, j^{\prime}\right),(j, i) \nsim\left(j^{\prime}, i^{\prime}\right)$ and $\left(j, i^{\prime}\right) \sim\left(j^{\prime}, i\right),\left(i^{\prime}, j\right) \sim\left(i, j^{\prime}\right)$. The pairs designated as non-matching have equal differences and the same sign.

Let $i, j$ be symmetric even blocks. Then $d\left(i, i^{\prime}\right)=d\left(j, j^{\prime}\right)=n, d(i, j)=d\left(i^{\prime}, j^{\prime}\right), d\left(j, i^{\prime}\right)=$ $d\left(j^{\prime}, i\right)$. Also $\left(i, i^{\prime}\right) \sim\left(j, j^{\prime}\right)$ if and only if $(i, j) \sim\left(i^{\prime}, j^{\prime}\right)$ if and only if $\left(j, i^{\prime}\right) \sim\left(j^{\prime}, i\right)$. Now $\left(i, i^{\prime}\right) \sim\left(j, j^{\prime}\right)$ if and only if the pairs have opposite signs. If $\left(i, i^{\prime}\right)$ is matched with $\left(j, j^{\prime}\right)$ then match $\left(j, i^{\prime}\right)$ with $\left(j^{\prime}, i\right)$ and match $(i, j)$ with $\left(i^{\prime}, j^{\prime}\right)$.

Say a pair $(i, j)$ is not balanced or is unbalanced if either $i$ or $j$ is not symmetric. Let $i$ be an even non-symmetric block if one such exists. Consider a non-balanced pair $(i, j)$. If $(i, j) \sim(j, k)$ for some $k$ then $(i, k)$ is a unbalanced pair and is matched elsewhere. Suppose $(i, j) \sim(k, i)$. When $k$ is not symmetric this gives an unbalanced pair $(k, j)$ which is matched elsewhere. When $k$ is symmetric two cases can arise. Firstly if $k=j^{\prime}$ then $\left(j, j^{\prime}\right)$, which has distance $n$, is matched by a pair $\left(l, l^{\prime}\right)$ for a symmetric $l$ and this gives a new unmatched pair $(i, l)$ which is not balanced and is matched elsewhere. Secondly if $k \neq j^{\prime}$, a new unbalanced pair $\left(i, k^{\prime}\right)$ is obtained which is matched elsewhere. Suppose $(i, j) \sim(k, l)$ where none of the indices are equal. Then $(i, k) \sim(j, l)$ match and $d(j, k) \neq d(i, l), d(j, k) \neq d(l, i)$ as $i$ is not symmetric. Hence $(i, l) \nsim(j, k)$ and $(i, l) \nsim(k, j)$. This gives the new unbalanced pair $(i, l)$ which has a match elsewhere. In all cases a new unbalanced pair is derived which must be matched elsewhere. Continue this matching process. However this cannot continue indefinitely. Thus if a a non-symmetric even 2-block exists there is a pair of even 2-blocks which is not matched. Hence every even block is symmetric. It follows that every odd block is symmetric.

Since all the blocks are symmetric, $n$ must be even and let $n=2 q$. Consider now the odd 2-blocks. These may then be listed as $\left\{i_{1}, i_{2}, \ldots, i_{q}, i_{1}^{\prime}, i_{2}^{\prime}, \ldots, i_{q}^{\prime}\right\}$, where $i_{j}$ means the block $B_{i_{j}}$ and the indices are ordered $i_{1}<i_{2}<\ldots<i_{q}$. Say $j \in T$ if $j$ is one of $\left\{i_{1}, i_{2}, \ldots, i_{q}\right\}$.

Suppose first of all that $n=4$. Then the odd 2-blocks are $\left\{i, j, i^{\prime}, j^{\prime}\right\}$. If $(i, j) \sim\left(i^{\prime}, j^{\prime}\right)$ then $\left(j, i^{\prime}\right) \nsim\left(j^{\prime}, i\right)$ and if $\left(j, i^{\prime}\right) \sim\left(j^{\prime}, i\right)$ then $(i, j) \not\left(i^{\prime}, j^{\prime}\right)$. This is impossible.

Suppose then $n>4$. For each $i, j \in T$ consider the pairs in $T(i, j)=\left\{i, j, i^{\prime}, j^{\prime}\right\}$ and match these where possible. As already noted $\left(i, i^{\prime}\right) \sim\left(i^{\prime}, i\right),\left(j, j^{\prime}\right) \sim\left(j^{\prime}, j\right)$. Either $(i, j) \sim\left(i^{\prime}, j^{\prime}\right)$ and $\left(j, i^{\prime}\right) \nsim\left(j^{\prime}, i\right)$ or else $\left(j, i^{\prime}\right) \sim\left(j^{\prime}, i\right)$ and $(i, j) \nsim\left(i^{\prime}, j^{\prime}\right)$. Call the non-matching pairs, $(i, j),\left(i^{\prime}, j^{\prime}\right)$ or $\left(j, i^{\prime}\right),\left(j^{\prime}, i\right)$ as appropriate, and their conjugates the remainders from $T(i, j)$ and denote these by $\operatorname{rem}(T(i, j))$. Other pairs from $T(i, j))$ besides those in $\operatorname{rem}(T(i, j))$ are matched. The remainders occur in pairs which have equal differences and same sign together with their conjugates which must also have equal differences and same sign. Clearly $T(i, j)=T(j, i))$ and so in specifying $T(i, j)$ we can assume from now on that $i<j$. For $i<j$ the elements in $\operatorname{rem}(T(i, j))$ with differences less than $n$ are either $(i, j),\left(i^{\prime}, j^{\prime}\right)$ or $\left(j, i^{\prime}\right),\left(j^{\prime}, i\right)$ and the other remainders are the conjugates of these with differences greater than $n$.

If one remainder from $T(i, j)$ matches a remainder from $T(k, l)$ then all the remainders from $T(i, j)$ can be matched with remainders from $T(k, l)$ and in this case say $\operatorname{rem}(T(i, j))$ match $\operatorname{rem}(T(k, l))$. If $\operatorname{rem}(T(i, j))$ match $\operatorname{rem}(T(k, l))$ then $\operatorname{rem}(T(k, l))$ match $\operatorname{rem}(T(i, j))$.

Suppose $\operatorname{rem}(T(i, j))$ match $\operatorname{rem}(T(j, k))$ or $\operatorname{rem}(T(k, i))$ for some $k$. In this case say $\operatorname{rem}(T(i, j))$ are matched by the remainders from an adjacent pair. Consider the case that $\operatorname{rem}(T(i, j))$ match $\operatorname{rem}(T(j, k))$; the other case is similar. Then $d(i, j)=d(j, k)$ and $\operatorname{rem}(T(i, k))$ match remainders from another pair. If $\operatorname{rem}(T(i, k))$ are matched by the remainders from an adjacent pair then this gives further remainders $\operatorname{rem}(T(i, r))$ or $\operatorname{rem}(T(r, k))$ matched by the remainders from some other $T(p, q)$. Continue in this way until eventually $T(p, q)$ is obtained whose remainders are not matched by remainders from an adjacent pair. 
Suppose $\operatorname{rem}(T(i, j))$ match $\operatorname{rem}(T(k, l))$ where $i, j, k, l$ are distinct and we may assume that $i<k$. Then $d(i, j)=d(k, l)$. (Note that $i<j, k<l$ and that $d(i, j)=d(k, l)$ if and only if $d\left(j, i^{\prime}\right)=d\left(l, k^{\prime}\right)$.) Then either $k<j$ or $k>j$; assume $k>j$ and the other case is similar. Then $d(i, k)=d(j, l)$ and $d(i, l)=d\left(k, j^{\prime}\right)$. It follows that either $\operatorname{rem}(T(i, l))$ match $\operatorname{rem}(T(j, k))$ and $\operatorname{rem}(T(i, k)), \operatorname{rem}(T(j, l))$ consist of two pairs from each with equal differences and same sign together with their conjugates or else $\operatorname{rem}(T(i, k))$ match $\operatorname{rem}(T(j, l))$ and $\operatorname{rem}(T(i, l))$, rem $(T(k, j))$ consist of two pairs from each with equal differences and same sign together with their conjugates. Match all remainders possible resulting from the match of $\operatorname{rem}(T(i, j))$ and $\operatorname{rem}(T(k, l))$. There still remains either $\operatorname{rem}(T(i, k))$ and $\operatorname{rem}(T(j, l))$ or $\operatorname{rem}(T(i, l))$ and $\operatorname{rem}(T(k, j))$ which are matched by remainders from different pairs. If these remainders can be matched by remainders from an adjacent pair this leads to a pair whose remainders cannot be matched by remainders of an adjacent pair as already pointed out. Continue matching in this way. At each stage a new pair is obtained whose remainders are matched by the remainders of another pair not already matched. This process cannot continue indefinitely and hence there is a pair of odd 2-blocks which is not matched.

Thus the matrix $M$ is not Hadamard for $n>1$ and thus the original matrix is not Hadamard as required. This completes the proof that there is no Hadamard $4 n \times 4 n$ matrix for $n>1$.

\section{Hadamard matrices from group rings}

We have shown that no circulant Hadamard matrices of size $4 n \times 4 n$ for $n>1$ can exist. Equivalently, there is no Hadamard $R G$-matrix from the group ring $\mathbb{Z} C_{4 n}$-matrix except when $n=1$. For the case $n=1$, we showed in (1.1) an example of a Hadamard matrix from the group ring $\mathbb{Z} C_{4}$.

A natural question arises: From other groups $G$, when do Hadamard matrices exist? Lemma 3.2 tells us that in general it is necessary for the group to be of order $4 u^{2}$.

We now provide a couple of illustrations of these matrices, first from groups of direct products of cyclic groups, and then using a direct product of the quaternion group with cyclic groups.

The following is a Hadamard $\mathbb{Z}\left(C_{2} \times C_{2}\right)$-matrix: $\left(\begin{array}{cc|cc}1 & 1 & 1 & -1 \\ 1 & 1 & -1 & 1 \\ \hline 1 & -1 & 1 & 1 \\ -1 & 1 & 1 & 1\end{array}\right)$.

In the cyclic (circulant) case a Hadamard matrix is not obtained because of a 'twist' in one of the pairs $(+,-),(-,+)$ from the end of one block to the beginning of the next. By avoiding this 'twist' a Hadamard matrix that is 'almost' circulant may be obtained.

Consider thus the case $n=16$ where the first row is

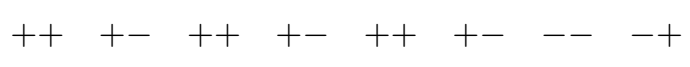


and then proceed by avoiding the 'twist' that a circulant would introduce to get the following Hadamard matrix:

$$
\left(\begin{array}{c|c|c|c|c|c|c|c}
++ & +- & ++ & +- & ++ & +- & -- & -+ \\
++ & -+ & ++ & -+ & ++ & -+ & -- & +- \\
\hline-+ & ++ & +- & ++ & +- & ++ & +- & -- \\
+- & ++ & -+ & ++ & -+ & ++ & -+ & -- \\
\hline-- & -+ & ++ & +- & ++ & +- & ++ & +- \\
-- & +- & ++ & -+ & ++ & -+ & ++ & -+ \\
\hline+- & -- & -+ & ++ & +- & ++ & +- & ++ \\
-+ & -- & +- & ++ & -+ & ++ & -+ & ++ \\
\hline++ & +- & -- & -+ & ++ & +- & ++ & +- \\
++ & -+ & -- & +- & ++ & -+ & ++ & -+ \\
\hline+- & ++ & +- & -- & -+ & ++ & +- & ++ \\
-+ & ++ & -+ & -- & +- & ++ & -+ & ++ \\
\hline++ & +- & ++ & +- & -- & -+ & ++ & +- \\
++ & -+ & ++ & -+ & -- & +- & ++ & -+ \\
\hline+- & ++ & +- & ++ & +- & -- & -+ & ++ \\
-+ & ++ & -+ & ++ & -+ & -- & +- & ++
\end{array}\right)
$$

The above example is actually a Hadamard $R G$-matrix from the group $\operatorname{ring} \mathbb{Z}\left(C_{2} \times C_{8}\right)$. This may be extended in an obvious way to form Hadamard $R G$-matrices from $\mathbb{Z}\left(C_{2} \times C_{8} \times C_{4}^{t}\right)$ for any $t . C_{4}$ may be replaced by $C_{2} \times C_{2}$ to give further examples.

Looking at how the twists work as in the proof of Theorem 1.1, a Hadamard $R G$-matrix from the group ring $\mathbb{Z}\left(\mathbb{H} \times C_{2}\right)$ may be constructed as follows, where $\mathbb{H}$ is the quaternion group of order 8 . This is an example of a Hadamard $R G$-matrix where $G$ is non-commutative.

$$
\left(\begin{array}{c|c|c|c||c|c|c|c}
++ & +- & ++ & +- & ++ & -+ & -- & +- \\
++ & -+ & ++ & -+ & ++ & +- & -- & -+ \\
\hline-+ & ++ & -+ & ++ & +- & ++ & -+ & -- \\
+- & ++ & +- & ++ & -+ & ++ & +- & -- \\
\hline++ & +- & ++ & -+ & -- & +- & ++ & +- \\
++ & -+ & ++ & +- & -- & -+ & ++ & -+ \\
\hline-+ & ++ & +- & ++ & -+ & -- & -+ & ++ \\
+- & ++ & -+ & ++ & +- & -- & +- & ++ \\
\hline++ & -+ & -- & +- & ++ & +- & ++ & +- \\
++ & +- & -- & -+ & ++ & -+ & ++ & -+ \\
\hline+- & ++ & -+ & -- & -+ & ++ & -+ & ++ \\
-+ & ++ & +- & -- & +- & ++ & +- & ++ \\
\hline-- & +- & ++ & +- & ++ & +- & ++ & -+ \\
-- & -+ & ++ & -+ & ++ & -+ & ++ & +- \\
\hline-+ & -- & -+ & ++ & -+ & ++ & +- & ++ \\
+- & -- & +- & ++ & +- & ++ & -+ & ++
\end{array}\right)
$$

There are 'twists' here which fit together to cancel one another. This may be extended to examples of Hadamard $R G$-matrices from $\mathbb{Z}\left(\mathbb{H} \times C_{2} \times \mathcal{C}_{4}^{t}\right)$ in an obvious way. $C_{4}$ may be replaced by $C_{2} \times C_{2}$ to give similar examples.

\section{References}

1. K. J. Horadam, Hadamard matrices and their applications (Princeton University Press, 2007).

2. T. Hurley, 'Group rings and rings of matrices', Int. J. Pure Appl. Math. 31 (2006), 319-335. 
3. H. Kharaghani and B. Tayfeh-Rezaie, 'A Hadamard matrix of order 428', J. Combin. Des. 13 (2005), 435-440.

4. C. Milies and S. Sehgal, An introduction to Group Rings (Klumer, Dordrecht, 2002).

5. H. J. Ryser, Combinatorial mathematics (Wiley, New York, 1963).

6. B. Schmidt, 'Cyclotomic integers and finite geometries', J. Amer. Math. Soc. 12 (1999) 929-952.

7. B. Schmidt, 'Towards Ryser's Conjecture', Proceedings of the Third European Congress of Mathematics (eds C. Casacuberta et al.), Progress in Mathematics 201 (Birkhuser, Boston, 2001) 533-541.

8. B. Schmidt, Characters and cyclotomic fields in finite geometry (Springer, Berlin, 2002).

9. R. Turyn, 'Character sums and difference sets', Pacific. J. Math. 15 (1965) 319-346.

Barry Hurley and Ted Hurley

National University of Ireland Galway

Galway

Ireland.

barry.hurley@nuigalway.ie

ted.hurley@nuigalway.ie

\section{Paul Hurley}

IBM Research Zurich

Säumerstrasse 4, CH-8803 Rüschlikon

Switzerland.

pah@zurich.ibm.com 